\title{
EXPORT: Optical photometry and polarimetry of Vega-type and pre-main sequence stars ${ }^{\star}$
}

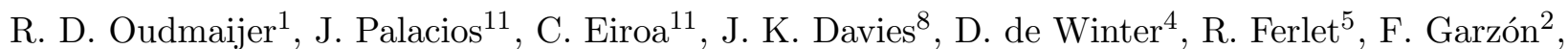
C. A. Grady ${ }^{6}$, A. Cameron ${ }^{7}$, H. J. Deeg ${ }^{3}$, A. W. Harris ${ }^{7,9}$, K. Horne ${ }^{7}$,

B. Merín ${ }^{10}$, L. F. Miranda ${ }^{3}$, B. Montesinos ${ }^{3,10}$, A. Mora ${ }^{11}$, A. Penny ${ }^{12}$, A. Quirrenbach ${ }^{13}$, H. Rauer ${ }^{9}$, J. Schneider ${ }^{14}$, E. Solano ${ }^{10}$, Y. Tsapras ${ }^{7}$, and P. R. Wesselius ${ }^{15}$

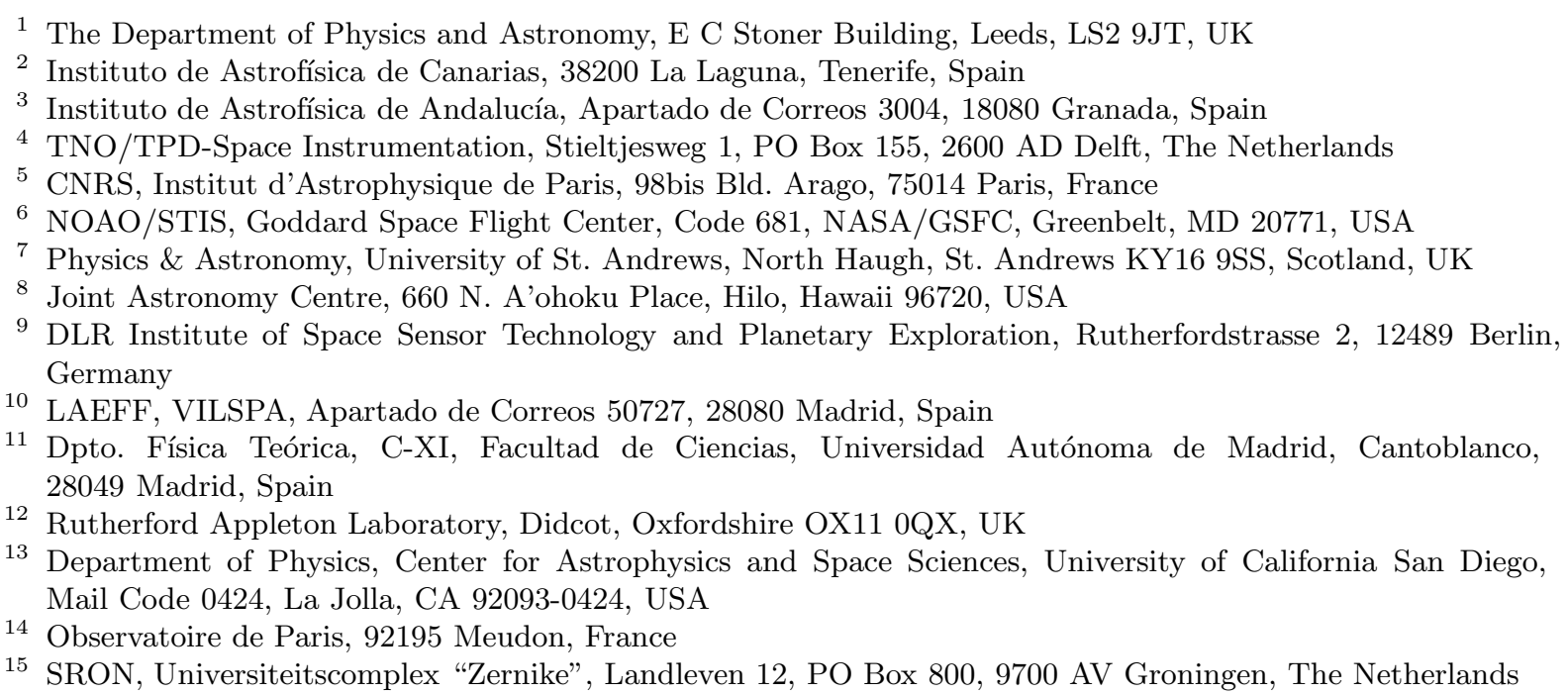

Received 6 August 2001 / Accepted 20 September 2001

\begin{abstract}
This paper presents optical UBVRI broadband photo-polarimetry of the EXPORT sample obtained at the $2.5 \mathrm{~m}$ Nordic Optical Telescope. The database consists of multi-epoch photo-polarimetry of 68 pre-mainsequence and main-sequence stars. An investigation of the polarization variability indicates that 22 objects are variable at the $3 \sigma$ level in our data. All these objects are pre-main sequence stars, consisting of both T Tauri and Herbig Ae/Be objects while the main sequence, Vega type and post-T Tauri type objects are not variable. The polarization properties of the variable sources are mostly indicative of the UXOR-type behaviour; the objects show highest polarization when the brightness is at minimum. We add seven new objects to the class of UXOR variables (BH Cep, VX Cas, DK Tau, HK Ori, LkH $\alpha$ 234, KK Oph and RY Ori). The main reason for their discovery is the fact that our data-set is the largest in its kind, indicating that many more young UXOR-type pre-main sequence stars remain to be discovered. The set of Vega-like systems has been investigated for the presence of intrinsic polarization. As they lack variability, this was done using indirect methods, and apart from the known case of $\mathrm{BD}+31^{\circ} 643$, the following stars were found to be strong candidates to exhibit polarization due to the presence of circumstellar disks: $51 \mathrm{Oph}, \mathrm{BD}+31^{\circ} 643 \mathrm{C}$, HD 58647 and HD 233517.
\end{abstract}

Key words. techniques: photometry - techniques: polarimetry - stars: circumstellar matter stars: pre-main sequence - stars: variables: general

Send offprint requests to: R. D. Oudmaijer,

e-mail: roud@ast. leeds.ac.uk

* Table A1 is only available in electronic form at the CDS via anonymous ftp to cdsarc.u-strasbg.fr (130.79.128.5) or via

http://cdsweb.u-strasbg.fr/cgi-bin/qcat?J/A+A/379/564

\section{Introduction}

One of the main projects within the EXPORT project (EXoPlanetary Observational Research Team, Eiroa et al. 2000) concerned the study of the circumstellar gas and dust around pre-main-sequence (PMS) and main-sequence 
(MS) objects thought to be accompanied by planets, or by planets in formation. The aim of this effort was to study stars at different early evolutionary stages by gathering more observational data on the existing samples in order to provide better defined observational clues regarding proto-planetary disk evolution. The data concerned are near-simultaneous spectroscopy, photometry and polarimetry. Such data are complementary, as, roughly speaking, the former data probe the gas closest to the stars, while the photo-polarimetry most likely probes gas and dust further out from the stars. Although studies of individual objects have been carried out in the recent past, a large sample of objects has not been studied simultaneously in a systematic and statistical manner. This is mainly due to the large amounts of telescope time needed - made possible only now by the allocation of the 1998 La Palma International Time to the EXPORT project. Near-infrared photometry of our sample has been published in Eiroa et al. (2001), while the first results on the spectroscopy are presented in Mora et al. (2001).

This paper concerns the presentation and preliminary analysis of the optical photo-polarimetry obtained in the course of the project. The purpose of these data is twofold, in the first place, the data are used to "flux" the spectroscopic data so that not only changes in the emission line equivalent widths can be traced, but also the energy output. Secondly, combined polarimetry and photometry teaches us a great deal about the geometry of the circumstellar material. Stellar light that is scattered off circumstellar dust grains will result in the light being polarized. In the case of spherically symmetric shells, or face-on circular disks, no net polarization will be observed as all polarization vectors cancel each other out. On the other hand, in the cases of a deviation from circular symmetry on the sky, a net polarization will be observed.

It is not trivial to assess whether a polarized object indeed has a disk-like dust configuration, as interstellar polarization can often contribute significantly to the observed polarization. Variability of the polarization can settle this question quite straightforwardly, as any variations in polarization must be due to circumstellar effects, because interstellar dust clouds are not expected to vary within the epochs under consideration.

A sub-sample of both low and intermediate mass young objects displays a special type of photo-polarimetric variability, commonly referred to as the "UX Ori" phenomenon (Grinin et al. 1994). From long term photopolarimetric monitoring of a small number of young stars, Grinin et al. (1994) identified a group of objects that are photo-polarimetrically variable. This group of stars shows increased polarization when the optical light of the stars is faint. Crucially, the objects also are redder at fainter magnitudes, while in extreme visual minima there is a colour reversal, the observed colours become bluer again. Named after their proto-type, UX Orionis, these stars are commonly referred to as UXORs. UX Ori itself is classified as a Herbig $\mathrm{Ae} / \mathrm{Be}$ star, and indeed many UXORs are on the more massive end of the young stellar mass spectrum.
Yet, this behaviour is not confined to Herbig stars, as for example the T Tauri object BM And does show the same behaviour (Grinin et al. 1995).

The main explanation of this phenomenon concerns the existence of dust clumps located in a disk-like configuration rotating around the star. When the dust clumps are not in our line of sight, the star will be observed at maximum light, with only a slight contribution of scattered radiation from the dust. If the dust is intersecting the line of sight, light from the star will be absorbed, and the relative contribution of the scattered light increases, increasing the observed polarization. Moreover, the fact that the reddening of the star coincides with the faintening, leaves little doubt that dust-absorption indeed plays the main role in the process. In cases of extremely deep minima, the light from the star is blocked almost entirely, resulting in a "blueing" of the energy distribution, as now mostly scattered light dominates the observed light. Depending on the distribution of dust-clouds, the light can be more or less absorbed during a period of photo-polarimetric monitoring. Added complications to this simplified scenario are aligned grains and dust production and destruction (see Grinin 1994). For more detailed discussions on the origins of UXOR photo-polarimetric behaviour, we refer the reader to Grinin (1994) and Grinin et al. (1994), but see Herbst \& Shevchenko (1999) who discuss some short-comings of the scenario. In the following, we will refer to stars exhibiting the UX Ori phenomenon when an increased polarization is observed simultaneously with fainter, and possibly redder, photometric results. We appear not to have observed objects in very deep minima, hence the "blueing effect" is not present in our data.

The aims of this paper are threefold. It is meant as a database for the community, and as such the dataacquisition, reduction and results are presented. In addition, the first analysis of the data is presented, aimed firstly at the detection of variability of the sources, and secondly at identifying Vega-type objects that may exhibit intrinsic polarization due to dusty circumstellar circumstellar disks.

This paper is organized as follows: Sect. 2 presents the observations and data reduction, Sect. 3 discusses the variability of the sources and Sect. 4 presents the analysis of the Vega-type objects, while we end with a discussion in Sect. 5.

\section{Source selection, observations and data-reduction}

\subsection{Source selection}

In order to learn more on the statistical behaviour of planetary systems, and especially their formation, the observed sample covers stars in various stages of their evolution. 
Low mass young stellar objects such as the Classical T Tauri stars (CTTs) and Early type T Tauri stars (ETT) were selected from Herbst et al. (1994). A sample of their intermediate mass counterparts, the Herbig Ae/Be stars, was taken from the Thé et al. (1994) catalogue. The observed sample covers almost all such stars observable in the Northern hemisphere. Objects further in their evolution, which are now on, or close to, the Main Sequence, were also included. This sub-sample includes Vega-type stars, MS stars that are found to exhibit an infrared excess identified with circumstellar, possibly protoplanetary, disks. The spectral types cover the low to intermediate mass range. These objects were mostly taken from Sylvester et al. (1996). Finally, some A-type shell stars found to be accreting gas, a spectral characteristic of the Vega-type object $\beta$ Pic, were taken from Grady et al. (1996). The list of targets and some results are presented in Table 1.

\subsection{Observations}

The observations employed the Turpol UBVRI polarimeter/photometer (Korhonen et al. 1984) mounted on the the $2.5 \mathrm{~m}$ Nordic Optical Telescope (NOT), La Palma, Spain. The data are obtained simultaneously in the $U B V R I$ bands (with equivalent wavelengths of $0.36,0.44$, $0.53,0.69$ and $0.83 \mu \mathrm{m}$ ) via several dichroic beamsplitters. A half-wave plate rotated in steps of $22.5^{\circ}$ enables linear polarization measurements to be made. One complete polarization measurement consists of eight integrations. The sky background polarization is eliminated by the use of the calcite block, so only sky intensity measurements are recorded, mostly before and immediately after a target observation.

The data were collected on photo-multipliers. Neutral density filters (1\% and $10 \%$ throughput respectively) were used to block the light from very bright sources. The use of these neutral density filters resulted in roughly the same number of photons per unit time being recorded from bright sources and faint sources. Hence, for the same exposure times, similar observational error bars on the resulting polarization and photometry are obtained for the targets. The objects have been observed with exposure times of $10 \mathrm{~s}$ (per integration) with 4 cycles giving total exposure times of $320 \mathrm{~s}$, with the exception of the photometric standards for which 1 cycle only was taken.

The observations were obtained during the nights of 14-17 May 1998, 28-31 July 1998, 23-27 October 1998 and 29-31 January 1999. All nights were of good enough quality to yield useful polarization data, while about half of the nights were photometric. Apart from July, in all runs a 10 arcsec diaphragm was used, in July the 7.5 arcsec diaphragm was employed. Calibration observations were taken throughout the nights, these include polarized standard stars, zero-polarization stars and photometric standards with different colours, observed at different air-masses.
Table 1. The observed objects. Columns 2 and 3 list the number of polarimetric and photometric observations respectively. Column 4 gives the weighted mean of the $V$ band polarization, as an indication of the average level of polarization observed, and finally, it is noted whether the polarization was found to be variable or not (see text).

\begin{tabular}{|c|c|c|c|c|}
\hline Name & $N_{\text {pol }}$ & $N_{\text {phot }}$ & $P_{\mathrm{V}}(\%)$ & Variable? \\
\hline \multicolumn{5}{|l|}{ Herbig Ae/Be: } \\
\hline AS 442 & 3 & 0 & $2.93 \pm 0.04$ & \\
\hline $\mathrm{BD}+40^{\circ} 4124$ & 4 & 3 & $1.17 \pm 0.03$ & \\
\hline HD 31648 & 6 & 5 & $0.40 \pm 0.02$ & \\
\hline HD 34282 & 3 & 3 & $0.12 \pm 0.02$ & \\
\hline HD 144432 & 7 & 3 & $0.25 \pm 0.02$ & \\
\hline HD 150193 & 3 & 2 & $4.64 \pm 0.02$ & \\
\hline HD 179218 & 1 & 1 & $0.64 \pm 0.07$ & \\
\hline HD 190073 & 3 & 1 & $0.41 \pm 0.03$ & \\
\hline $\mathrm{LkH} \alpha 234$ & 8 & 5 & $0.44 \pm 0.07$ & $\checkmark$ \\
\hline MWC 297 & 1 & 1 & $1.15 \pm 0.17$ & \\
\hline VX Cas & 9 & 7 & $0.69 \pm 0.03$ & $\checkmark$ \\
\hline SV Cep & 10 & 6 & $1.03 \pm 0.02$ & $\checkmark$ \\
\hline V1686 Cyg & 11 & 7 & $3.14 \pm 0.07$ & $\checkmark$ \\
\hline VY Mon & 3 & 2 & $9.82 \pm 0.23$ & $\sqrt{ }$ \\
\hline $51 \mathrm{Oph}$ & 6 & 3 & $0.47 \pm 0.02$ & \\
\hline KK Oph & 9 & 3 & $5.26 \pm 0.05$ & $\checkmark$ \\
\hline BF Ori & 6 & 4 & $0.46 \pm 0.01$ & $\sqrt{ }$ \\
\hline T Ori & 6 & 4 & $0.29 \pm 0.02$ & \\
\hline UX Ori & 9 & 6 & $1.15 \pm 0.01$ & $\checkmark$ \\
\hline V346 Ori & 5 & 3 & $0.22 \pm 0.02$ & \\
\hline V350 Ori & 5 & 3 & $2.57 \pm 0.05$ & $\checkmark$ \\
\hline XY Per & 6 & 5 & $1.58 \pm 0.01$ & $\sqrt{ }$ \\
\hline VV Ser & 11 & 7 & $1.72 \pm 0.04$ & \\
\hline RR Tau & 7 & 5 & $1.38 \pm 0.03$ & $\checkmark$ \\
\hline WW Vul & 11 & 7 & $0.27 \pm 0.02$ & $\sqrt{ }$ \\
\hline \multicolumn{5}{|c|}{ Herbig/ZAMS: } \\
\hline HD 58647 & 3 & 1 & $0.16 \pm 0.02$ & \\
\hline HD 141569 & 9 & 4 & $0.62 \pm 0.02$ & \\
\hline HD 142666 & 7 & 3 & $0.71 \pm 0.04$ & \\
\hline HD 163296 & 7 & 3 & $0.02 \pm 0.01$ & \\
\hline HD 199143 & 2 & 2 & $0.11 \pm 0.04$ & \\
\hline \multicolumn{5}{|l|}{ T Tauri: } \\
\hline HD 123160 & 5 & 2 & $0.30 \pm 0.04$ & \\
\hline BM And & 8 & 7 & $2.03 \pm 0.06$ & $\checkmark$ \\
\hline BH Cep & 11 & 8 & $0.44 \pm 0.03$ & $\checkmark$ \\
\hline BO Cep & 9 & 6 & $0.88 \pm 0.03$ & \\
\hline CO Ori & 8 & 6 & $2.22 \pm 0.03$ & $\checkmark$ \\
\hline HK Ori & 7 & 6 & $1.18 \pm 0.03$ & $\sqrt{ }$ \\
\hline NV Ori & 7 & 5 & $0.58 \pm 0.01$ & $\checkmark$ \\
\hline RY Ori & 7 & 5 & $2.62 \pm 0.05$ & $\checkmark$ \\
\hline CQ Tau & 7 & 5 & $0.20 \pm 0.01$ & $\checkmark$ \\
\hline CW Tau & 8 & 7 & $0.79 \pm 0.12$ & $\sqrt{ }$ \\
\hline DK Tau & 5 & 5 & $1.31 \pm 0.07$ & $\checkmark$ \\
\hline DR Tau & 4 & 4 & $0.46 \pm 0.06$ & \\
\hline RY Tau & 7 & 6 & $2.62 \pm 0.02$ & $\checkmark$ \\
\hline $\mathrm{PX}$ Vul & 9 & 6 & $3.89 \pm 0.03$ & \\
\hline \multicolumn{5}{|l|}{ Vega/PTT: } \\
\hline $\mathrm{BD}+31^{\circ} 643$ & 8 & 6 & $1.26 \pm 0.03$ & \\
\hline $\mathrm{BD}+31^{\circ} 643 \mathrm{C}$ & 3 & 2 & $0.70 \pm 0.03$ & \\
\hline HD 23362 & 3 & 1 & $0.66 \pm 0.04$ & \\
\hline HD 23680 & 2 & 1 & $1.18 \pm 0.06$ & \\
\hline HD 34700 & 2 & 2 & $0.35 \pm 0.06$ & \\
\hline HD 109085 & 1 & 1 & $0.05 \pm 0.04$ & \\
\hline HD 142764 & 3 & 2 & $1.56 \pm 0.03$ & \\
\hline HD 233517 & 3 & 1 & $1.64 \pm 0.03$ & \\
\hline HR 26 & 1 & 1 & $0.09 \pm 0.06$ & \\
\hline HR 419 & 3 & 1 & $0.19 \pm 0.06$ & \\
\hline HR 1369 & 1 & 1 & $0.03 \pm 0.05$ & \\
\hline HR $1847 \mathrm{~A}$ & 3 & 3 & $0.63 \pm 0.04$ & \\
\hline HR $1847 \mathrm{~B}$ & 3 & 3 & $0.63 \pm 0.02$ & \\
\hline HR 2174 & 3 & 1 & $0.07 \pm 0.03$ & \\
\hline HR $2174 \mathrm{~B}$ & 1 & 0 & $0.07 \pm 0.04$ & \\
\hline HR $4757 \mathrm{~B}$ & 1 & 0 & $0.11 \pm 0.09$ & \\
\hline HR $5422 \mathrm{~A}$ & 4 & 2 & $0.08 \pm 0.02$ & \\
\hline HR 5422B & 4 & 2 & $0.06 \pm 0.04$ & \\
\hline HR 9043 & 3 & 3 & $0.04 \pm 0.04$ & \\
\hline$\lambda$ Boo & 4 & 1 & $0.03 \pm 0.01$ & \\
\hline $49 \mathrm{Cet}$ & 2 & 0 & $0.15 \pm 0.06$ & \\
\hline \multicolumn{5}{|l|}{ A-shell: } \\
\hline $24 \mathrm{CVn}$ & 3 & 1 & $0.02 \pm 0.02$ & \\
\hline $17 \mathrm{Sex}$ & 3 & 1 & $0.07 \pm 0.03$ & \\
\hline HR 10 & 3 & 3 & $0.07 \pm 0.04$ & \\
\hline
\end{tabular}




\subsection{Data reduction}

The polarization data were reduced using software designed for the Turpol, both RDO and JP (using a similar programme) reduced the data independently, and the results were similar. $1 \sigma$ error bars are calculated from least-square fits to the data in the eight different orientations of the retarder, or the photon noise, whichever is the largest. The resulting accuracy in the $\boldsymbol{Q}$ and $\boldsymbol{U}$ Stokes vectors is of order $0.05-0.1 \%$ for the sources in most bands. The error in the polarization is the same as the error in the Stokes vectors. Typical errors on the polarization angle were of order $1-3^{\circ}$ and calculated from $0.5 \times \operatorname{atan}\left(\sigma_{P} / P\right)$, which, to first approximation equals the commonly adopted $28.65^{\circ} \times \sigma_{P} / P^{1}$. In one instance, during the first two observations of 29 January 1999, a problem with the field rotator occurred, resulting in an error in the position angle that is hard to quantify. For these two objects (BD+31643 and HD 23362), we set the error in PA to $10^{\circ}$. It should be noted here that the computed error-bars reflect the statistical, internal, error-bars. The external errors are expected to be slightly larger, of order $0.1 \%$, as polarization standard stars often have slightly different published values, possibly due to different filterbands and systems used. We also find deviations at the $1-2^{\circ}$ level when intercomparing polarized standards. In those cases we took the weighted mean of the correction to be applied. This adds an additional uncertainty to the derived position angles of $1-2^{\circ}$, which does not affect the results in the vast majority of cases.

The instrumental polarization as determined from observations of zero-polarization stars, was mostly below $0.05 \%$ and thus not of relevance to the data reported here. The system was extremely stable during the four runs, statistical tests, outlined below, showed that highly polarized standard stars were recorded with the same polarization throughout, which was consistent with values from the literature. The zero-polarized stars remained constant within the error bars, indicating that the instrumental polarization was constant at low values.

We note that measured polarizations, by virtue of the way they are calculated $\left(P=\sqrt{Q^{2}+U^{2}}\right)$, are always positive, and hence at the low polarization end, the data are inevitably biased towards too high polarization values. The usual manner of dealing with such data is correcting in the manner $P_{\text {true }}=\sqrt{P_{\text {obs }}^{2}-0.5 \pi \sigma_{P}^{2}}$ (cf. Serkowski 1962). Here, we will not correct for this bias and not subtract the instrumental polarization from the resulting data (mostly due to pragmatic reasons as the objects often have

1 These approximations do not strictly hold for very low polarization values (see Serkowski 1962, 305), but only for $P \gg 0 \%$, or, roughly, $P \geq 6 \sigma_{P}$. For $P \sim 0 \%$, the errors on the polarization are actually smaller than the error bars on $\boldsymbol{Q}$ and $\boldsymbol{U}$ by about $35 \%$, while the error bars on the polarization angle reach a maximum of $52^{\circ}$. These values are related to the complex form of error-propagation - see Serkowski $(1962,304)$ and further. larger polarizations), but it should be kept in mind when investigating low polarization targets.

Nine out of the 16 nights were photometric, these were 15 and 16 May, 29 July and 23-27 October 1998, and 30 January 1999. The photometry was reduced using standard procedures, and taking into account atmospheric extinction. It was found that the neutral density (ND) filters were not entirely grey, and additional corrections had to be performed on the final values. The problem stemmed from the fact that the observed Landolt standard stars were mostly faint $(V>9)$, and no ND filter was needed, while some bright target stars needed ND filters in order to avoid burning the photo-multipliers. The corrections were determined by comparing existing photometry, taken from SIMBAD, and the results were as follows: for the $10 \% \mathrm{ND}$ filter no correction was needed in the $U B V R$ bands, while the correction amounted to -0.15 mag in the $I$ band. The $1 \%$ ND filter had a larger effect; $-0.08 \mathrm{mag}(V)$, $-0.18 \mathrm{mag}(R)$ and $-0.45 \mathrm{mag}$ in the $I$ band, while the $U$ and $B$ bands did not need a correction. The error-bars on the photometry were dominated by the solutions to the colour transformation rather than shot-noise statistics, and are less than 0.1 mag overall. Conservative limits of $0.10,0.10$ and $0.15 \mathrm{mag}$ are adopted for the $V, R$ and $I$ band data respectively where corrections had to be applied because of the 1\% ND filter and $0.10 \mathrm{mag}$ for the $I$ band in the case of the $10 \%$ ND filter.

In total, 68 targets were observed. We attempted to observe every star at least twice during each observing run, and during consecutive observing runs. The resulting data set thus contains photo-polarimetric monitoring on daily and monthly time-scales with most of the objects having been observed $5-10$ times. The resulting data set is provided in the Appendix, which is available electronically at CDS.

\section{Results}

Although a detailed analysis of the data is beyond the scope of this paper, we discuss some global properties of the sample below. The main property that we discuss first is the presence of polarimetric variability - which confirms the presence of a flattened structure around the objects. We then discuss the polarization characteristics of the Vega-type stars and identify targets for follow-up research.

\subsection{Variability of the sources}

In this section we will discuss the variability of the sources. Determining whether the sources are variable is not necessarily straightforward as outlined in the previous footnote. The error propagation from the $\boldsymbol{Q} \boldsymbol{U}$ vectors is not trivial, complicating statistical tests on the resulting polarization and angle. Hence, we have to perform a test in $\boldsymbol{Q U}$ space.

To this end, we adopt the simple, yet powerful statistical formalism presented by Oudmaijer \& Bakker (1994) 

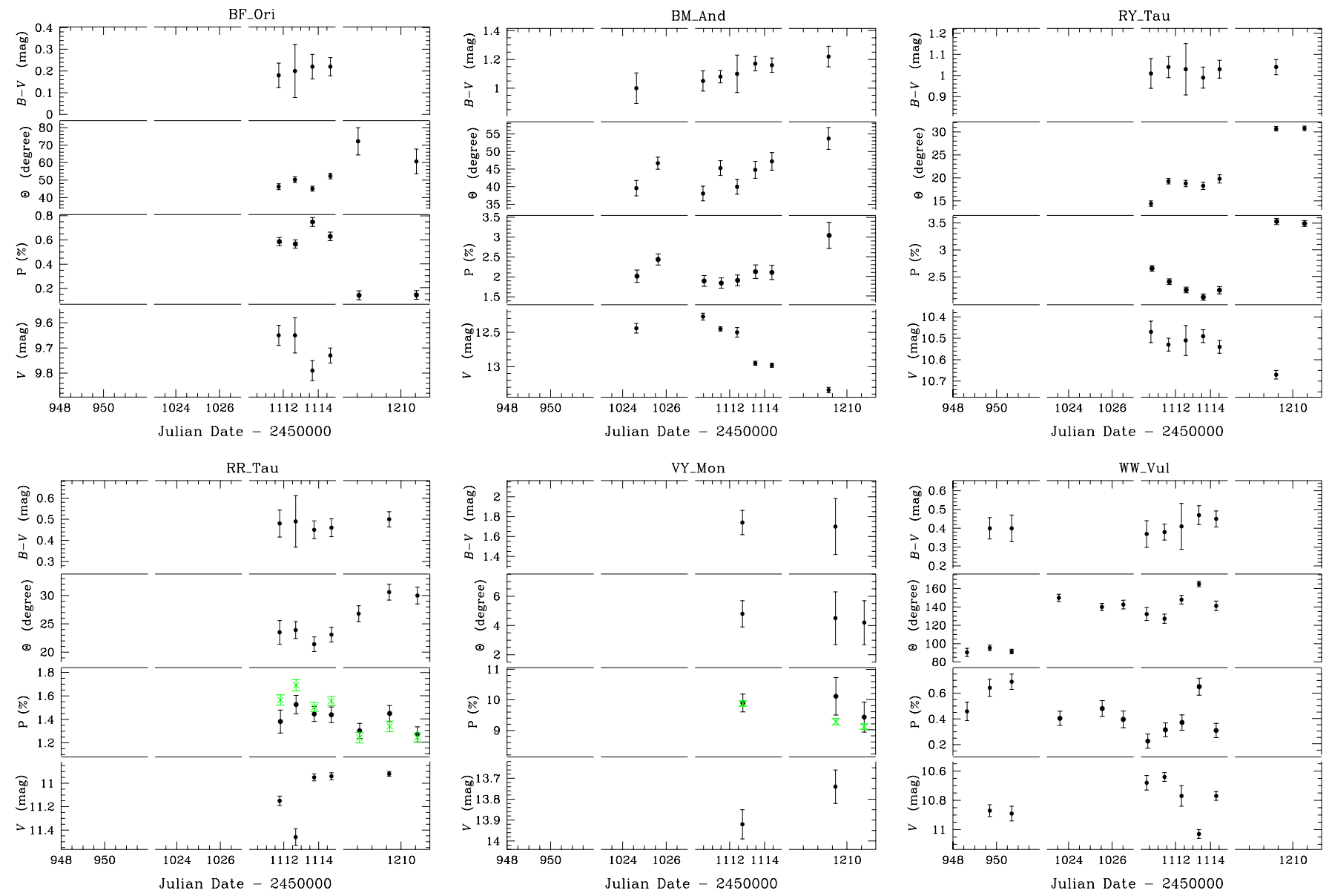

Fig. 1a. Objects known to exhibit the UXOR phenomenon and showing it in our data. Plotted are the $(B-V)$, Position Angle, Polarization and $V$ magnitude as function of time. For selected objects (see text), the crosses denote the $R$ band polarization values. The four panels in Julian Date correspond to the May, July and October 1998 and January 1999 observing runs.

and Henrichs et al. (1994). The variability can be expressed as

$$
\left(\frac{\sigma_{\mathrm{obs}}}{\sigma_{\mathrm{av}}}\right)_{\lambda} \approx \sqrt{\frac{1}{N-1} \sum_{i=1}^{N}\left(\frac{P_{i}(\lambda)-P_{\mathrm{av}}(\lambda)}{\sigma_{i}(\lambda)}\right)^{2}} .
$$

Where $\sigma_{\mathrm{obs}}$ represents the scatter of the data-points around the mean observed value, $\sigma_{\mathrm{av}}$ is the (average) experimental error, $N$ is the number of data points, $P_{\text {av }}(\lambda)$ represents the (weighted) average Stokes parameters, $P_{i}(\lambda)$ the individual Stokes parameters, and $\sigma_{i}(\lambda)$ is the $1 \sigma$ error-bar for the data points.

$\left(\sigma_{\mathrm{obs}} / \sigma_{\mathrm{av}}\right)$ corresponds to the standard deviation of the variations of the individual data points with respect to the average divided by the standard deviation of the average. If no significant variations are present, the value will be close to one, significant deviations are directly represented in units of the noise level, that is to say a peak of three corresponds to a variability at a $3 \sigma$ level. This method gives a numerical answer to the question: is the rms of the mean of all observations larger than the individual error-bars? The method was applied on the individual $\boldsymbol{Q}$ and $\boldsymbol{U}$ vectors for every photometric band of the observed targets. The mean value was close to 1 , confirming that the error-bars provided by the reduction software reflect the true errors. A more stringent confirmation of the strength of this statistical test is that the polarization and photometric standard stars appeared not to be variable at a level larger than $2 \sigma$. An exception to this finding was the polarized standard HD 154445. This star was flagged as variable at the $3 \sigma$ level in almost all photometric bands. Inspection of the data revealed that this was due to the star being very close to the bright limits of the photo-multipliers for its ND filter, which resulted in more counts than recorded for the average target. As such this star has very low experimental error bars, sometimes at the $0.03 \%$ and $0.3^{\circ}$ level, so that systematic uncertainties in polarization angles can not be ignored anymore (see the discussion in the previous section).

We selected all objects that displayed variations at the $>3 \sigma$ level in either the $\boldsymbol{Q}$ or $\boldsymbol{U}$ Stokes vector in at least 1 band. These objects are indicated in Table $1^{2}$.

${ }^{2}$ Four objects that satisfied this criterion are not listed in the table. The statistical results of HD 144432, HD 163296 and V346 Ori were dominated by one deviant polarization value in only one band in the sequence of observations, which was probably related to the low polarization of the sources. These stars are not further discussed here. $\tau$ Boo is effectively unpolarized in our data, with the listed polarizations of order the observational error-bars. 

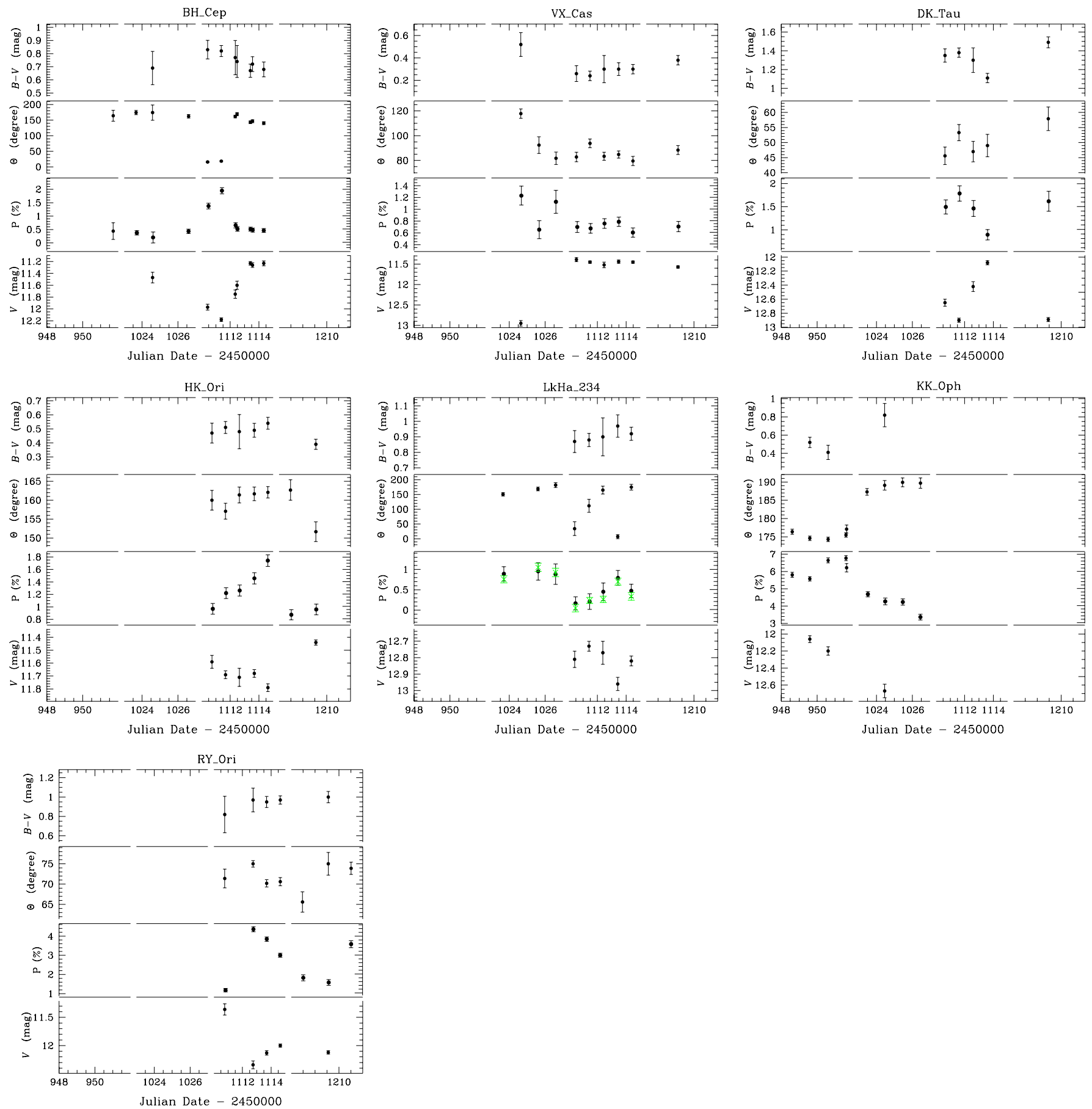

Fig. 1b. As the previous figure, but now for objects not previously known to show the UXOR behaviour.

Virtually all T Tau stars were found to be variable, while half of their earlier-type counterparts, the Herbig Ae/Be stars are variable in our data-set. There are undoubtedly more variable stars in the sample, as for example low level but periodic variability will not be recognized as such using the above procedure. A detailed investigation however is beyond the scope of this paper.

\subsection{Correlation photometry - polarization}

For the objects flagged as variable in Table 1, we investigate the correlation between the observed polarization and photometry. From here on, we will discuss them into two groups: those objects showing increased polarization with fainter magnitudes (i.e. showing the UXOR phenomenon), and those objects that do not clearly show this behaviour in our dataset. We base the following on the $V$ band; where necessary, other passbands will be discussed as well. We point out that the following discussion is not exhaustive as not all observing dates were photometric, limiting the number of the combined photo-polarimetric data. It is also possible that our limited time-coverage may have missed objects that are known to be variable, but happened to be relatively stable during our observing campaigns. 


\subsection{Objects showing increased polarization with fainter magnitudes}

In our sub-sample of 22 targets which display significant variations, the UXOR type behaviour (in first instance, an anti-correlation between polarization and photometry) can be found in 13, i.e. more than half of the targets. Their data are displayed in Figs. 1a and b. For six of these (BF Ori, BM And, RR Tau, VY Mon, WW Vul and RY Tau), similar observations are present in the literature, while for seven of these objects (BH Cep, KK Oph, RY Ori, VX Cas, DK Tau, HK Ori and LkH $\alpha 234)$ this is a new finding, as, to our knowledge, no previous photopolarimetric monitoring has been performed for these objects.

BF Ori: the UXOR behaviour was previously discussed by Grinin et al. (1989) for BF Ori. We seem to have observed the star in a relatively bright period, $V<10$ $(P<1 \%)$. Grinin et al. (1989) observed minima with $V>$ 12 , with $P$ reaching the $5 \%$ level.

$B M$ And, RY Tau, and WW Vul: for both BM And (Grinin et al. 1995) and WW Vul (Grinin 1994) a similar behaviour as for BF Ori has been presented in the literature. At some times, polarizations of order $6 \%$ were measured when the stars were at their photometric minima ( $V=14$, and 12 respectively). We observed these stars in relatively bright, longer lasting, phases. RY Tau was observed by Petrov et al. (1999), in a somewhat brighter, lower polarization state than reported here.

$R R$ Tau: although variable in the entire polarization data-set, the variability of RR Tau is less significant in the subset containing the photometry. The minimum in the $V$ band photometry corresponds to a hardly significant local maximum in polarization but is more visible in the $R$ band data, which are also shown for this object. RR Tau is known to display the UXOR phenomenon, as Rostopchina et al. (1997) has shown. They find that the $V$ band magnitude varies between 10 and 14, with excursions in the polarization up to $6 \%$.

VY Mon: the subset containing both polarimetry and photometry of VY Mon is limited to only two data-points. As the errors on the $V$ band polarization are large, the $R$ band data are plotted in the figure as well. In the $V$ band there is no polarization variability at all, but in $R$ and $I$ bands the UXOR phenomenon becomes clearly visible. Because of the faintness and redness of the object, the observational error bars prevent us from detecting variability in $V$ - a problem eased by investigating the $R$ band, where the UXOR phenomenon becomes visible. Based on two unpublished photometric data-points, Yudin \& Evans (1998) reach the same conclusion.

We now add to this class of object an additional seven stars. Although the photometric variability of these objects was known already, as is evident from their names of course, it is our simultaneous photo-polarimetric campaign that puts them in the "UXOR" class.
BH Cep: BH Cep shows photometric variations of $1 \mathrm{mag}$, coinciding with a ten-fold polarization increase from 0.2 to $2 \%$ within two days.

$V X$ Cas: a slight anti-correlation is seen in the October 1998 run, the single point from July shows a deep brightness minimum accompanied by high polarization and the reddest colour.

DK Tau: shows a 1 mag change and a doubling of the polarization from 1 to $2 \%$ within two days.

HK Ori: displays a gradual change over a four day period by 0.4 mag while increasing in polarization from $1 \%$ to $2 \%$.

LkH人234: exhibits a drop of 0.3 mag in 1 day, coinciding with an increase in polarization from 0.1 to $0.7 \%$. This is more clearly present in the $R$ band data.

$K K O p h$ : only three photometric points are available for this highly variable object. Within the May 1998 run, the two data points show an anti-correlation between photometry and polarimetry, indicating UXOR type behaviour. The third data point, taken in July 1998, however is the faintest photometric point, but also shows the lowest polarization. It may well be that the dust clouds responsible for the obscuration in July 1998 had a different geometry (i.e. less a-symmetric) resulting in lower polarization values and a different inclination, as evidenced by the change in position angle between the two runs.

$R Y$ Ori: the October 1998 run displays a very clear UXOR behaviour, a change of 1 mag in brightness is accompanied by a polarization increase of $4 \%$. As for the previous object, this is not followed during the next run, where the polarization has decreased, while the $V$ band magnitude remained constant.

\subsection{Objects not showing increased polarization with fainter magnitudes in our data}

Here, we discuss the nine stars which, from our preliminary analysis, appear to show no anti-correlation between polarization and brightness or for which we can make no conclusive remarks. In some cases the objects are mostly variable in the photometry, but the errors on the $V$ band polarization prevent us from detecting variability - the variability being traced in the entire polarization data-set but not in the subset containing the photometry. However, using additional data-points, or judging from information in the literature, we find that by and large these objects are most likely to show the UXOR phenomenon as well. The objects that appear to show a positive correlation between polarization and magnitude, rather than an anti-correlation, do so when different observing epochs are compared. This could well be explained within the UXOR scenario if one assumes that different dust-clouds are responsible for the respective polarization and obscuration. 
SV Cep: SV Cep's polarization variability has decreased substantially in the subset containing the photometry, which is probably why our data do not show the UXOR phenomenon. A recent paper by Rostopchina et al. (2000) presents 10 year long monitoring of the star, and only one deep minimum was detected. Indeed. the polarization increased during this event, suggesting SV Cep is also a member of the UXOR class. It is plausible that the rareness of the polarimetric events is the reason why we missed the effect altogether.

V350 Ori: the subset of V350 Ori containing the photometry with only three data points, does show significant polarization variations. Here, the observational errors on the photometry make an assessment of the variations inconclusive. Interpreting their own, sparse, data of the object, Yudin \& Evans (1998) claim that it shows the UXORtype behaviour, but it is unclear whether this conclusion is based on simultaneously obtained photometry.

CO Ori: the photometric data of CO Ori show a gradual decrease in brightness over five days in October 1998, while the polarization data show a slight, but not significant, increase in polarization. When the star is at its faintest detected level, it exhibits the lowest polarization - contrary to the usual UXOR behaviour. This "deviating" point is from another epoch (January 1999), a period when the observed polarization was consistently lower than in October 1998, but with only one photometric night available. As for KK Oph and RY Ori discussed above, it may well be that the dust clouds responsible for the obscuration in January 1999 had a different geometry. If true, then CO Ori is another object to be added to the UXOR category.

NV Ori: during the October 1998 run, the data of NV Ori cluster within the errors bars around $V \sim 9.8$, while in polarization small changes are detected. As for CO Ori, in the subsequent observing epoch, both the polarization and optical brightness have decreased dramatically, If the object was obscured, but relatively quiescent in October, and a different cloud is causing the obscuration in January 1999, it is well possible that NV Ori is a new member of the UXOR class.

$C Q$ Tau: as can be seen in Fig. 2, CQ Tau becomes redder with fainter $V$ band magnitudes, but the polarization is a minimum in the middle of the photometric range. An effect also seen in the $B$ band (not shown). A clue to CQ Tau's behaviour is provided by the fact that we can see that the $R$ (shown in the figure) and $I$ band polarization behave exactly as expected from an UXOR variable.

It is interesting to note that the change in polarization is more clearly detectable at longer wavelengths. Although this is counter-intuitive, since polarization increases with shorter wavelengths, this is not a new result. Inspection of the figures in e.g. Grinin et al. (1991), shows that this indeed happens in some cases. The possible reason is that we are looking at the polarization vector addition of the circumstellar polarization and interstellar polarization. Since both can have a different wavelength dependence, the (vector-)addition of the two will show a different wavelength dependence. The change in PA from the first two days in October to the latter two is dramatic. In the case of one of the two components varying, we will then also see a varying total component. In principle, in the photometric minimum of the star, the circumstellar polarization should dominate, while in photometric maximum the interstellar component, depending on its magnitude, can dominate.

Grinin (1994) pointed out that CQ Tau does show the UXOR phenomenon, but with some exceptions, sometimes when the star has larger polarization, it is brighter.

$C W$ Tau: for convenience, the $R$ band data are also shown for this object, as the faintness of the star prevents us from discussing the lower quality $V$ band data. CW Tau shows photometric variations in excess of $1.5 \mathrm{mag}$, but, with the exception of one higher polarization point in both $V$ and $R$ remains quite constant in polarization. It was especially the changes in Position Angle that were picked up by the variability-software, and we only note at this stage that the interplay between circumstellar and interstellar polarization may play a role for this object. Clearly, CW Tau deserves further study.

UX Ori: it is ironic that UX Orionis should be classified as not displaying the UXOR behaviour. There is no obvious trend visible in the data, but the range of $V$ band photometry is the smallest observed in the sample of polarimetrically variable objects, with variations less than 0.25 mag. Comparison with data of Grinin et al. (1994) reveals that we have caught the object in a bright, low polarization spell. The changes in polarization are accompanied with significant changes in PA, indicating that several polarization mechanisms may be acting on the object. We previously discussed the interplay between circumstellar dust polarization and the interstellar polarization, however, we point out that low amplitude photometric variations do not have to necessarily be due to the dust obscuration phenomenon alone. As shown for example by Oudmaijer \& Drew (1999), electron-scattering can contribute up to $1 \%$ to the total polarization towards Herbig Be stars. If the excitation conditions within the ionized envelope change, slight changes in polarization can be readily detected.

$X Y$ Per and V1686 Cyg: despite the comparatively large data coverage for these two stars, we are not in a position to classify the stars' behaviour during our observations. Although the stars show photometric variations of more than $1.5 \mathrm{mag}$, and relatively strong polarization variability it is hard to describe a trend.

Of the nine objects discussed in this section, we find that four objects (CQ Tau, UX Ori, SV Cep, V350 Ori) had previously been reported to be UXOR variable stars. These stars do not show the expected anti-correlation between polarimetry and photometry in our data. The reason is most likely that our subset containing the photometric data was too small to find this behaviour as well. 

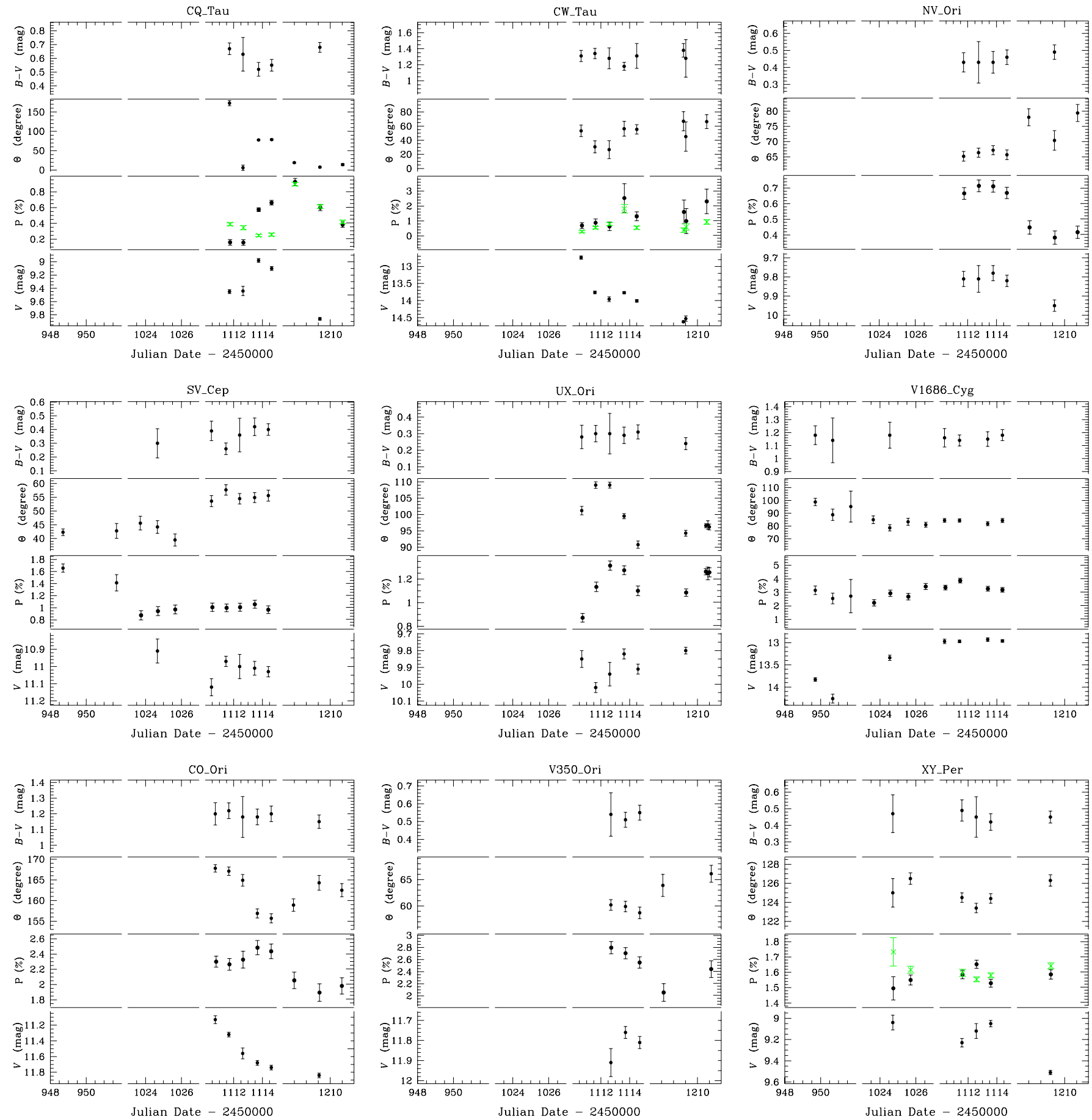

Fig. 2. As the previous figures, but now for objects that do not seem to exhibit the UXOR behaviour. The open squares in the figures for VY Mon and XY Per denote the $I$ band data.

For two objects (CO Ori, NV Ori) we find opposed behaviour to the expected anti-correlation. Their behaviour can be incorporated into the explanation of the UXOR phenomenon, provided different dust clouds act on the polarization, as was found for example in the case of KK Oph.

For the remaining three objects (CW Tau, V1686 Cyg and XY Per), we are not able to classify their behaviour, and further studies are warranted.

\subsection{Conclusion on the polarization variability}

From the 22 objects in our data-set that showed significant variability, most of them appear to display the UXOR phenomenon i.e. show increased polarization when fainter. This is a rather loose definition of the UXOR phenomenon, as the colour changes play a central role in the explanation of the phenomenon as well. However, on the whole, we have not detected the strong photometric changes of more than 4 mag with their associated colour changes of 
order $0.3-0.5 \mathrm{mag}$. This is most likely due to the fact that more often than not the UXOR variables are in their bright state, preventing us from detecting their more extreme behaviour in our comparatively short monitoring programme.

Some objects, which are variable in their entire polarization dataset, were not variable in the subset containing the photometry. In those cases, existing literature on these objects showed that they are UXOR variables.

Indeed, most of the objects known as UXOR variable, were recovered via our statistical tests. A notable exception is VV Ser (Kardopolov et al. 1991) in which variations are observed, but not in such a way that they satisfy our criteria outlined in Sect. 3. It is most likely our comparatively sparse sampling that prevents us from catching all such objects, but on the whole the detection rate has been very high.

We add a total of seven new objects to the class of UXORs, while, if we were to include NV Ori and CO Ori, showing photo-polarimetric variability as well, we increase the sample by nine. These objects were previously not recognized as such because they had not been photopolarimetrically monitored before.

\section{Polarization properties of the Main Sequence objects}

In this section, we discuss the polarization properties of the main sequence stars in our sample. The sample comprises Vega-type stars, A shell type stars and binary systems consisting of an early type main sequence primary and a late secondary classified as post-T Tauri star. In some of the latter cases, only the primary was observed. We further included some Herbig Ae/Be stars that are known to be located very close to the main sequence and $51 \mathrm{Oph}$ that is sometimes classified as a Herbig Ae/Be star and sometimes listed as Vega-type.

For many of these stars, listed in Table 2, we present the first polarization measurements, and for virtually all of these stars photo-polarimetric monitoring has been performed for the first time.

The main question that we will address here is whether these objects have intrinsic polarization due to the presence of a stellar disk. As mentioned in the Introduction only observed variability in the polarization of an object presents strong evidence for the presence of intrinsic polarization. However, as shown above, only the PMS stars show a high degree of variability, hence we will try to answer the question of the presence of intrinsic polarization via different routes. Not all of these methods are conclusive, or as strong as the variability-test, but they will at least provide us with a sample of interest.

\subsection{The relation between observed polarization and extinction}

It is a rather well established finding that the interstellar polarization (ISP), which arises from the presence of aligned dust grains in the interstellar medium is correlated with the extinction due to the same dust grains. For example Serkowski et al. (1975) showed that the maximum polarization (in \%) is always smaller than $9 \times E(B-V)$ (in magnitudes). In fact, there is a relatively strong correlation between the observed polarization and extinction in such a way that $P_{\mathrm{V}}(\%) \sim A_{\mathrm{V}}$. For example, the sample of 361 field stars observed by Reiz \& Franco (1998, excluding two objects that have no derived reddening values) gives an average $\left(P_{\mathrm{V}}-A_{\mathrm{V}}\right)$ of 0.09 with a scatter of 0.23 . The scatter is larger than the estimated errors on the measured polarizations and derived reddenings by Reiz \& Franco.

Hence, a useful test would be to look for sources that have excess polarization compared to that expected from their observed $A_{\mathrm{V}}$. The polarization could then very well be due to a dusty disk, which does not contribute to the extinction, as an inclined disk would let the observer look straight at the star. To this end, we calculated the reddening to each of our objects, using our $(B-V)$ photometric data, spectral types taken from Mora et al. (2001), who obtained spectral types from spectra taken simultaneously with the photo-polarimetry in our EXPORT programme, and assuming a normal reddening law $A_{\mathrm{V}}=3.1 E(B-V)$. $E(B-V)$ was calculated using intrinsic $(B-V)$ colours listed in Schmidt-Kaler (1982) for the spectral types. For those objects that were not spectrally typed by EXPORT, or for which no photometry was available, the data provided by SIMBAD were used. The results are listed in Table 2, along with weighted mean values for the polarization and polarization angles in the $V$ band. The estimated error in $A_{\mathrm{V}}$ is about $0.2 \mathrm{mag}$, a result from the propagation of errors in the photometry (assumed to be $0.05 \mathrm{mag}$ for each band for each star) and the uncertainty in the spectral type (mostly two subclasses - Mora et al.) which results in an average uncertainty in $(B-V)_{0}$ of about $0.08 \mathrm{mag}$. The uncertainty in the luminosity class was not taken into account, only for $G$ and $K$ stars this would be larger.

The observed polarization as function of the derived $A_{\mathrm{V}}$ is shown in Fig. 3, where the relation $P_{\mathrm{V}}(\%) \sim A_{\mathrm{V}}$ is shown as a solid line, and the dotted lines represent a $3 \sigma$ error bar in this relation ( $0.6 \mathrm{mag}-$ see above). Most of the stars in the sample indeed fall within these boundaries, in fact, if we exclude the objects that fall either above or below these lines, we find an average $P_{\mathrm{V}}-A_{\mathrm{V}}$ of 0.05 with a root-mean-square scatter of 0.19 , very close to what is found for Reiz \& Franco's (1998) data.

The "excess" polarization of HR 5422B $\left(P_{\mathrm{V}}=0.06 \pm\right.$ $0.04 \%$ ) stands out because of its very negative $A_{\mathrm{V}}$ value. Inspection of the photometry shows that the data quality is good, we note that the spectral type should be changed from $\mathrm{K} 0$ to $\mathrm{G} 0$ in order to arrive at a different intrinsic $B-V$ colour or change the observed $B-V$ to 0.85 to alleviate this problem. The spectrum of the object shows hints of veiling (Mora, private communication) which may conspire with a possible UV-excess to result in too blue observed colours for its spectral type. 
Table 2. The Vega-type and related objects in our sample. Spectral types are taken from Mora et al. (2001) or from SIMBAD (marked with "S"). The photometry is taken from this paper, of from SIMBAD (marked with "S"). The polarization values are weighted means, also from this paper. $A_{\mathrm{V}}$ is derived from the spectral types and photometry, and the intrinsic $(B-V)$ values listed in Schmidt-Kaler (1982). $\lambda_{\max }, K$, and $\chi_{\text {red }}^{2}$ result from fitting Serkowski laws through the data (see text). The "types" are, unless otherwise noted, Vega-type.

\begin{tabular}{|c|c|c|c|c|c|c|c|c|c|c|}
\hline Name & $\begin{array}{l}\text { Spectral } \\
\text { Type }\end{array}$ & $\begin{array}{l}B \\
( \pm 0.05)\end{array}$ & $\begin{array}{l}V \\
( \pm 0.05)\end{array}$ & $\begin{array}{l}P_{\mathrm{V}} \\
(\%)\end{array}$ & $\begin{array}{l}\sigma_{P_{\mathrm{V}}} \\
(\%)\end{array}$ & $\begin{array}{r}A_{\mathrm{V}} \\
( \pm 0.2)\end{array}$ & $\begin{array}{l}\lambda_{\max } \\
(\mu \mathrm{m})\end{array}$ & 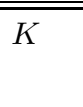 & 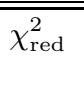 & type \\
\hline $17 \mathrm{Sex}$ & $\mathrm{A} 0 \mathrm{~V}$ & 5.94 & 5.92 & $<0.10$ & & 0.12 & & & & A-shell \\
\hline $24 \mathrm{Cvn}$ & $\mathrm{A} 4 \mathrm{~V}$ & 4.81 & 4.66 & $<0.05$ & & 0.12 & & & & A-shell \\
\hline 49 Cet & $\mathrm{A} 4 \mathrm{~V}$ & $5.68^{S}$ & $5.62^{S}$ & $<0.18$ & & -0.16 & & & & \\
\hline $51 \mathrm{Oph}$ & B9.5IIIe & 4.82 & 4.83 & 0.47 & 0.02 & 0.12 & 0.35 & 1.15 & 4.8 & Herbig Ae/Be/Vega \\
\hline BD 31643 & $\mathrm{~B} 5 \mathrm{~V}$ & 9.27 & 8.56 & 1.27 & 0.03 & 2.73 & 0.80 & 0.95 & 0.6 & \\
\hline BD $31643 \mathrm{C}$ & $\mathrm{A} 3 \mathrm{IV}^{S}$ & 11.18 & 10.3 & 0.70 & 0.03 & 2.45 & 0.90 & 0.15 & 3.5 & \\
\hline HD 23362 & K5III & 9.72 & 8.1 & 0.66 & 0.04 & 0.37 & 0.48 & 0.57 & 0.85 & \\
\hline HD 23680 & G5IV & 9.84 & 8.46 & 1.18 & 0.06 & 1.89 & 0.72 & 0.62 & 0.49 & \\
\hline HD 34700 & G0IV & 9.73 & 9.14 & 0.35 & 0.06 & -0.06 & 0.30 & 0.50 & 1.13 & \\
\hline HD 58647 & B9IV: & 6.92 & 6.85 & 0.16 & 0.02 & 0.43 & 0.30 & 0.02 & 2.11 & $\mathrm{H} / \mathrm{ZAMS}$ \\
\hline HD 109085 & $\mathrm{~F} 2 \mathrm{~V}$ & 4.66 & 4.32 & $<0.12$ & & -0.03 & & & & \\
\hline HD 123160 & K5III & 10.22 & 8.69 & 0.30 & 0.04 & 0.09 & 0.55 & 1.52 & 0.08 & \\
\hline HD 141569 & $\mathrm{~A} 0 \mathrm{~V}$ & 7.2 & 7.11 & 0.62 & 0.02 & 0.34 & 0.65 & 1.22 & 1.25 & $\mathrm{H} / \mathrm{ZAMS}$ \\
\hline HD 142666 & $\mathrm{~A} 8 \mathrm{~V}$ & 9.16 & 8.68 & 0.71 & 0.04 & 0.71 & 0.90 & 0.32 & 0.33 & $\mathrm{H} / \mathrm{ZAMS}$ \\
\hline HD 142764 & $\mathrm{~K} 7 \mathrm{~V}$ & 11.32 & 9.56 & 1.56 & 0.03 & 1.33 & 0.50 & 1.05 & 0.07 & \\
\hline HD 163296 & $\mathrm{~A} 1 \mathrm{~V}$ & 6.92 & 6.86 & $<0.04$ & 0.02 & 0.16 & & & & H/ZAMS \\
\hline HD 199143 & $\mathrm{~F} 6 \mathrm{~V}$ & 7.77 & 7.23 & $<0.13$ & & 0.19 & & & & H/ZAMS \\
\hline HD 233517 & $\mathrm{~K} 0 \mathrm{~V}$ & 11.03 & 9.69 & 1.64 & 0.03 & 1.64 & 0.32 & 0.18 & 2.02 & \\
\hline HR 10 & $\mathrm{~A} 0 \mathrm{~V}$ & 6.36 & 6.24 & $<0.12$ & & 0.43 & & & & A-shell \\
\hline HR 26 & B9V & 5.48 & 5.45 & $<0.17$ & & 0.31 & & & & $\mathrm{PTT} / \mathrm{MS}$ \\
\hline HR 419 & $\mathrm{~B} \mathrm{~V}^{S}$ & 6.6 & 6.65 & 0.19 & 0.06 & 0.06 & & & & $\mathrm{PTT}$ \\
\hline HR 1369 & $\mathrm{~B} 9 \mathrm{~V}^{S}$ & 5.31 & 5.38 & $<0.15$ & & 0.0 & & & & PTT \\
\hline HR 1847A & $\mathrm{B} 5 \mathrm{~V}$ & 6.12 & 6.11 & 0.63 & 0.04 & 0.56 & 0.58 & 0.85 & 0.51 & $\mathrm{PTT} / \mathrm{MS}$ \\
\hline HR 1847B & ${\mathrm{B} 7 \mathrm{IV}^{S}}^{S}$ & 6.52 & 6.48 & 0.63 & 0.02 & 0.53 & 0.64 & 0.62 & 0.18 & $\mathrm{PTT}$ \\
\hline HR 2174 & $\mathrm{~A} 2 \mathrm{~V}:$ & 5.8 & 5.73 & $<0.10$ & & 0.06 & & & & $\mathrm{PTT} / \mathrm{MS}$ \\
\hline HR 2174B & $\mathrm{A} 0^{S}$ & $6.9^{S}$ & $6.85^{S}$ & $<0.13$ & & 0.22 & & & & $\mathrm{PTT}$ \\
\hline HR 4757B & $\mathrm{K} 2 \mathrm{~V}$ & $9.46^{S}$ & $8.51^{S}$ & $<0.27$ & & 0.12 & & & & PTT \\
\hline HR 5422A & $\mathrm{A} 0 \mathrm{~V}$ & 6.04 & 6.09 & 0.08 & 0.02 & -0.09 & 0.59 & 1.98 & 0.12 & $\mathrm{PTT} / \mathrm{MS}$ \\
\hline HR 5422B & K0V & 11.93 & 11.37 & $<0.13$ & & -0.78 & & & & PTT \\
\hline HR 9043 & $\mathrm{~A} 5 \mathrm{~V}$ & 6.44 & 6.27 & $<0.12$ & & 0.06 & & & & \\
\hline$\lambda$ Boo & A1V & 4.26 & 4.22 & $<0.04$ & & 0.09 & & & & \\
\hline
\end{tabular}

We also find three objects which show less polarization than expected, $\mathrm{HD} 23680, \mathrm{BD}+31^{\circ} 643$, and $\mathrm{BD}+31^{\circ} 643 \mathrm{C}$. This would not be exceptional, as the observed relation between polarization and extinction should be regarded as an upper limit, but it could reveal the presence of a second polarization mechanism, destructively adding to the total polarization due to interstellar dust.

\subsection{Deviations from Serkowski law}

Although it can now be argued that all objects only suffer ISP as they are located on the expected relation between ISP and interstellar extinction, our multiwavelength data can offer ways to assess whether intrinsic polarization does contribute a fraction to the observed polarization.

We therefore continued with the sub-sample of Vegalike stars that are polarized, and removed objects with 


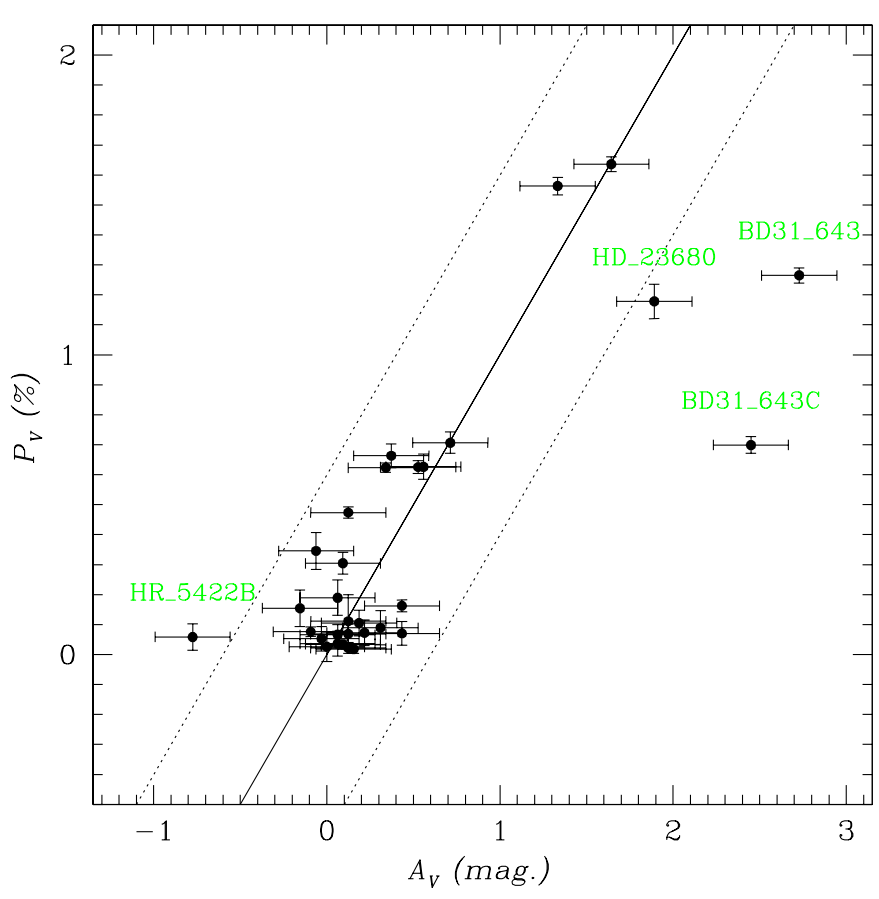

Fig. 3. Observed polarization in the $V$ band of the Vega-type stars plotted against their derived $A_{\mathrm{V}}$. The solid line represents the relation $P_{\mathrm{V}}(\%) \sim A_{\mathrm{V}}$, while the parallel dotted lines represent $3 \sigma$ error bars on the derived $A_{\mathrm{V}}$.

observed polarization less than the $3 \sigma$ level at more than one band. These stars are indicated with $3 \sigma$ upper limits in Table 2. HR 419 was only observed to be polarized in the $V$ band, and is also removed for the further study.

\subsubsection{The polarization angle as function of wavelength}

A first indication is to investigate whether the polarization angle, $\theta$, changes over the different pass bands. Normal interstellar polarization always displays the same position angle, as the grains responsible are aligned in the same manner. Any deviations from this may reveal the presence of an intrinsic component. We calculated, in much the same way as we detected variability in the polarization the rms scatter of the polarization angle at each wavelength around the mean value. This was then compared with the experimental error, set at minimally $0.5^{\circ}$ in line with the systematic errors expected. Two objects have a variability, computed using Eq. (1), in their polarization angles much larger than three. These are $\mathrm{BD}+31^{\circ} 643$ and $\mathrm{BD}+31^{\circ} 643 \mathrm{C}$.

\subsubsection{Wavelength dependence of polarization}

A further clue as to whether the observed polarization may be due to an (additional) intrinsic component is to check whether the data can be realistically fitted with a Serkowski law. As is well known, the wavelength dependence of ISP can be empirically expressed as

$p_{\lambda}=p_{\max } \exp \left[-K \ln ^{2}\left(\lambda_{\max } / \lambda\right)\right]$ with $p_{\max }$ the maximum polarization occurring at wavelength $\lambda_{\max }$, and $K$ describes the width of the Serkowski relation, originally set by default at 1.15 , it is now commonly used as a variable in parameterizing the broad-band polarization behaviour of stars (see e.g. Whittet et al. 1992 and 2001 for a thorough investigation on the subject). Normal interstellar material has $\lambda_{\max }$ in the range between 4000 and $8000 \AA$ and $K$ ranges between 0.5 and 1.5. Roughly speaking, $\lambda_{\max }$ traces the dominant size of the polarizing dust grains, while $K$ traces the width of the grain-size distribution. Deviations from this observed law may also indicate the presence of an intrinsic component, and this is what we investigate here. We therefore fitted the data of the polarized Vega-type objects with this Serkowski law, and searched the parameter space for the best fit, we let $\lambda_{\max }$ run from 3000 to $9000 \AA$ and $K$ from 0 to 2 . The results of these fits are plotted in Fig. 4 and also presented in Table 2 .

Based on the reduced $\chi^{2}$ computed, we immediately see that despite having generous limits for searching within $\lambda_{\max }$ and $K$ parameter space, bad fits were obtained for $51 \mathrm{Oph}, \mathrm{BD}+31^{\circ} 643 \mathrm{C}$, and to a lesser extent HD 58647 and HD 233517 which have reduced $\chi^{2}$ slightly larger than 2. It should also be noted that the best fits of all 4 objects were reached on the borders of the $\left(\lambda_{\max }, K\right)$ values explored, implying that the wavelength dependence of their polarization can not be due to normal interstellar material. The latter is also found for other objects, e.g. HD $142666\left(\lambda_{\max }=9000 \AA, K=0.32\right)$, however the small formal $\chi^{2}$ (due to the larger observational error bars), provides leeway for such objects to be satisfactorily represented by a more common combination. We note that the presence of two differently polarizing interstellar dust clouds might also result in a rotation or a different appearance of the polarization, however, the chances of this are probably slim, as the Serkowski law was derived from observations of such stars in the first place.

\subsection{Discussion on Vega-type stars}

Fifteen objects in our sample of 31 Vega-like systems exhibit, within our error bars, polarization. From simple tests on their polarization properties, we find that for most objects the observed polarization can be explained as normal interstellar polarization. We find evidence that five of these may have an intrinsic component in their polarization. These stars are discussed individually below:

$B D+31^{\circ} 643: \mathrm{BD}+31^{\circ} 643$ shows a trend in polarization angle which decreases by about $10^{\circ}$ from the $U$ band to $I$. This was noted already by Andersson \& Wannier (1997), who interpret this as an additional contribution of Rayleigh-scattered light from the circumstellar disk to the ISP.

$B D+31^{\circ} 643 C$ : this object is located less than 0.4 arcmin from $\mathrm{BD}+31^{\circ} 643$, and is also located within the IC 348 nebula. Little is known about this bright $(V \sim$ 10) object. Being picked up by all three of our diagnostic 

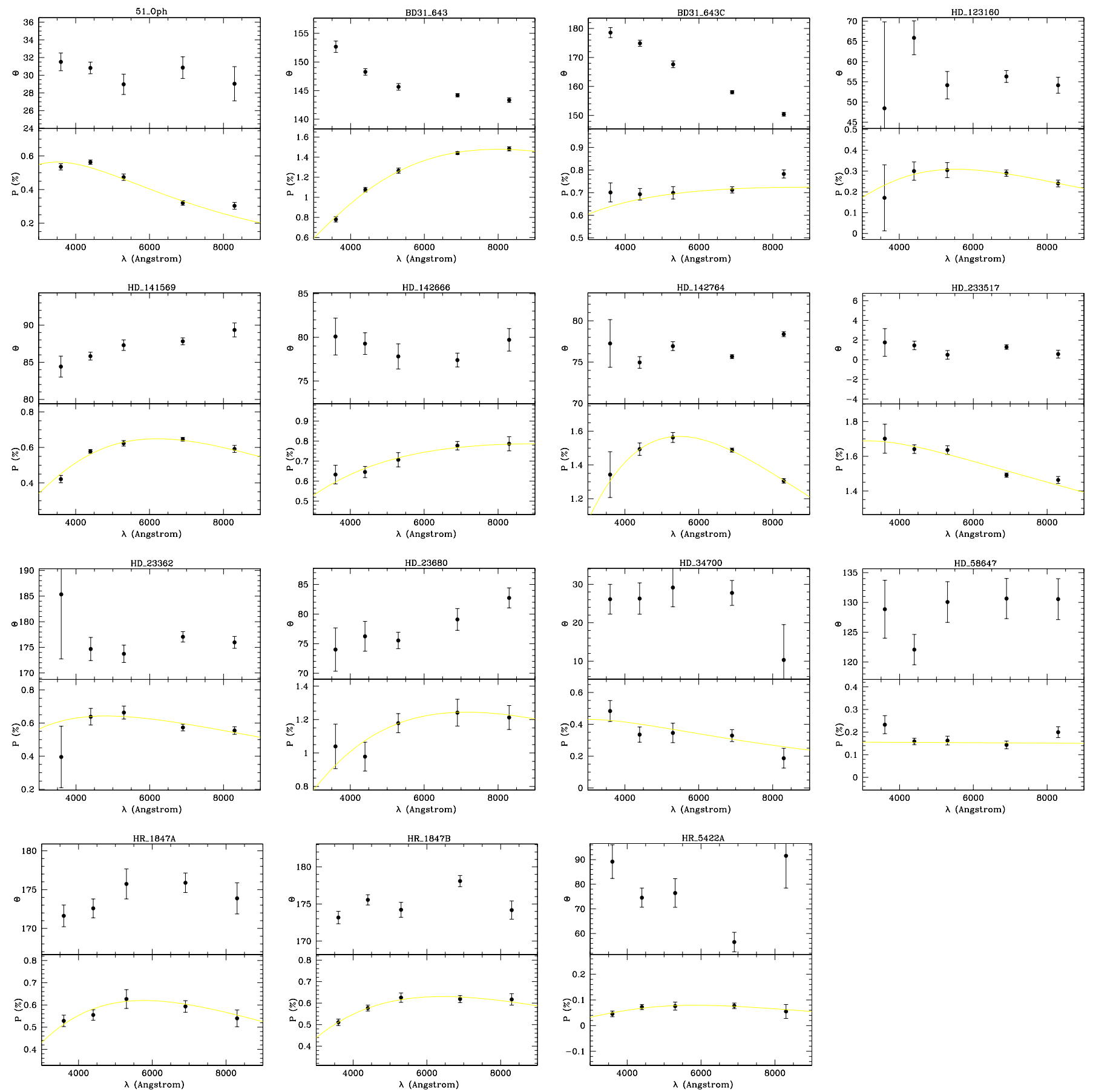

Fig. 4. Weighted mean values of the Polarization and PA as function of wavelength for the Vega-type stars in our sample. The solid lines denote the best fit Serkowski laws through the points (see text).

tests, our data show unambiguously that the polarization of this object can not be due to simple interstellar polarization alone. The different polarization behaviour of this star, being almost flat as opposed to the polarization of $\mathrm{BD}+31^{\circ} 643$ that peaks at long wavelengths, leaves us room to speculate. Assuming the objects were formed in the same cloud collapse, the later spectral type of $\mathrm{BD}+31^{\circ} 643 \mathrm{C}$ indicates a lower mass, and hence an earlier phase of formation. Contrary to $\mathrm{BD}+31^{\circ} 643$, the polarization of $\mathrm{BD}+31^{\circ} 643 \mathrm{C}$ does not fall off towards shorter wavelengths, implying that the smallest dust grains have not yet been removed during the star+disk evolution.

$51 \mathrm{Oph}$ : it is especially the increase in polarization towards smaller wavelengths that indicates this object to exhibit intrinsic polarization. Taken at face value, the higher polarization at shorter wavelengths indicates the presence of very small grains, consistent to the inferences 
made by van den Ancker et al. (2001) from the spectral energy distribution and features in the ISO-SWS spectrum. The polarization data clearly imply that the dust responsible for the infrared emission is located in the scattering disk.

HD 5864\%: this object stood out in the Serkowski fitting technique because of the apparent "excess" polarization in the $U$ and $I$ bands respectively. This behaviour was found on all three occasions we observed the object. As is the case for $51 \mathrm{Oph}$, HD 58647 is an $\mathrm{H} \alpha$ emitting object, and the possibility that electron-scattering plays a role can not be excluded. In this case the deviation from the Serkowski law would not imply a dusty disk-like structure but a disk-like structure in the ionized gas surrounding the object.

HD 23351\%: also has its polarization peak at extremely short wavelengths. Often included in studies of Vega-type objects, it now seems that HD 233517 actually is one of the K-giants with Lithium (Balachandran et al. 2000). The infrared excess of such giants has been interpreted being due to the evaporation of comets surrounding a Main Sequence star as it evolves off the Main Sequence (e.g. Plets et al. 1997). Our data indicate that these comet-like bodies may also be distributed in a disk. We note in passing that recently, not only HD 233517 has been identified as a Li-rich K-giant with infrared-excess instead of a Vega-type star, but also HD 23362 (Castilho et al. 1998), which was originally selected from existing lists of Vega-type stars as well.

\section{Discussion and concluding remarks}

We have observed a large sample of low and medium mass main sequence Vega-type stars and pre-main sequence objects photo-polarimetically over short (days) and long (months) time-scales. For many of these objects, these are the first such data obtained in this manner.

The main global property that we investigated here was the polarimetric variability of the objects- which confirms the presence of a flattened circumstellar dusty structure. The presence of variability was investigated in the data using relatively simple statistical methods. The objects classified as UXORs showed significant variability, both on short and long timescales. In most cases, the polarimetry is anti-correlated with the photometry: the objects show larger polarization when they are fainter. This is generally interpreted in terms of aspherically distributed dust clouds orbiting the star. These give rise to more extinction in the line-of-sight, while the scattered light will not be blocked. This results in an increase of the relative contribution of the scattered light to the total observed light, giving rise to larger polarization (e.g. Grinin et al. 1994).

Arguably, the distinction between "UXORs" (T Tauri and Herbig Ae/Be stars showing large variations in photometry and polarization) and "normal" $\mathrm{T}$ Tauri and Herbig Ae/Be stars becomes less pronounced based on results like these (see also Herbst \& Shevchenko 1999 for a discussion on photometric variability). Indeed, this large data-set indicates that the majority of the observed Herbig Ae/Be stars show evidence for non-spherical envelopes. Although there may have been a certain personal bias in the selection of the targets, it should be noted that most of the observable (northern) known Herbig Ae/Be stars have been observed in this project. Considering that the number of Herbig Ae/Be stars is only about one hundred (Thé et al. 1994), we speculate at this point that perhaps all massive PMS objects have aspherical structures. This is in line with the random orientations of any disks in the line of sight. If the disks are circular, then those disks oriented face-on would not show any polarization/polarization variability while projected rotational velocities would, in these cases, be lower. This is in contrast to van den Ancker et al. (1998), who did not find a correlation between photometric variations alone as a function of rotational velocity of Herbig Ae/Be stars.

Combining our results and the models of Natta \& Whitney may shed some light on this apparent problem. Natta \& Whitney (2000) argue that if Herbig Ae stars are surrounded by flaring dusty disks, the UXOR phenomenon can only be observed for a specific range of inclination angles. As outlined above, objects oriented face-on would not exhibit polarization variability. In addition, objects whose disks are heavily inclined would not be optically visible, due to the high optical depth in the disk mid-plane. Hence, objects with the largest observed projected rotational velocities are not included in any optically based sample of Herbig Ae/Be stars, limiting the velocity range probed by van den Ancker et al. Depending on the (unknown) opening angle of the dusty disk we may even miss a significant proportion of existing intermediate mass premain sequence stars. It may be worthy of note that Natta \& Whitney predict that around half of the optically visible Herbig Ae stars show the UXOR behaviour, just as observed here.

The combination of both photometric and polarimetric variability strongly suggests that all Herbig Ae/Be stars are surrounded by disks. Of course, we need to analyze the data further, but such a conclusion, albeit tentative, illustrates the importance of observing a large sample of PMS stars to be able to reach general conclusions on the properties of this evasive class of object.

The main result for the more than 30 Vega-type objects is that many are not polarized to our sensitivity, and those for which polarization is detected are not variable to within the error bars. Based on simple arguments, we have flagged 5 objects that may exhibit intrinsic polarization of which one $\left(\mathrm{BD}+31^{\circ} 643\right)$ was previously known, and list these as suitable candidates for future research.

Acknowledgements. The Nordic Optical Telescope is operated on the island of La Palma jointly by Denmark, Finland, Iceland, Norway, and Sweden, in the Spanish Observatorio del Roque de los Muchachos of the Instituto de Astrofísica de Canarias. 


\section{References}

Andersson, B.-G., \& Wannier, P. G. 1997, ApJ, 491, L103

Balachandran, S. C., Fekel, F. C., Henry, G. W., \& Uitenbroek, H. 2000, ApJ, 542, 978

Castilho, B. V., Gregorio-Hetem, J., Spite, F., Spite, M., \& Barbuy, B. 1998, A\&AS, 127, 139

Eiroa, C., (EXPORT) 2000, in Disks, Planetesimals and Planets, ed. F. Garzón, C. Eiroa, D. de Winter, \& T. Mahoney, ASP Conf. Ser., 219, 3

Eiroa, C., et al. (EXPORT) 2001, A\&A, 365, 110

Grady, C. A., Pérez, M. R., Talavera, A., et al. 1996, ApJ, 471, L49

Grinin, V. P. 1994, in The Nature and Evolutionary Status of Herbig Ae/Be stars, ed. P. S. Thé, M. R. Pérez, \& E. P. J. van den Heuvel, ASP Conf. Ser., 62, 63

Grinin, V. P., Kiselev, N. N., \& Minikulov, N. Kh. 1989, SvA Lett., 15, 1028

Grinin, V. P., Kiselev, N. N., Minikulov, N. Kh., Chernova, G. P., \& Voschinnikov, N. V. 1991, Ap\&SS, 186, 283

Grinin, V. P., Thé, P. S., de Winter, D., et al. 1994, A\&A, 292, 165

Grinin, V. P., Kolotilov, E. A., \& Rostopchina, A. 1995, A\&AS, 112,457

Henrichs, H., Kaper, L., \& Nichols, J. S. 1994, A\&A, 285, 565

Herbst, W., \& Shevchenko, V. S. 1999, AJ, 118, 1043

Herbst, W., Herbst, D. K., Grossman, E. J., \& Weinstein, D. 1994, AJ, 108, 1906

Kardopolov, V. I., Pavlova, V. I., \& Rspaev, F. K. 1991, AZh, 68,565

Korhonen, T., Piirola, V., \& Reiz, A. 1984, The ESO Messenger, December 1984

Mora, A., et al. (EXPORT) 2001, A\&A, 378, 116
Natta, A., \& Whitney, B. A. 2000, A\&A, 364, 633

Oudmaijer, R. D., \& Bakker, E. J. 1994, MNRAS, 271, 615

Oudmaijer, R. D., \& Drew, J. E. 1999, MNRAS, 305, 166

Petrov, P. P., Zajtseva, G. V., Efimov, Y. S., et al. 1999, A\&A, 341,553

Plets, H., Waelkens, C., Oudmaijer, R. D., \& Waters, L. B. F. M. 1997, A\&A, 323, 513

Reiz, A., \& Franco, G. A. P. 1998, A\&AS, 130, 133

Rostopchina, A. N., Grinin, V. P., Okazaki, A., et al. 1997, A\&A, 327, 145

Rostopchina, A. N., Grinin, V. P., Shakhovskoi, D. N., Thé, P. S., \& Minikulov, N. K. 2000, Astron. Reports, 44, 365

Schmidt-Kaler, Th. 1982, in Landolt-Börnstein, Numerical Data and Functional Relationships in Science and Technology, New Series Group VI, vol. 2h (Springer Verlag) Serkowski, K. 1962, in Adv. Astron. Astroph. 1, 289, 289

Serkowski, K., Mathewson, D. S., \& Ford, V. L. 1975, ApJ, 196,261

Sylvester, R. J., Skinner, C. J., Barlow, M. J., \& Mannings, V. 1996, MNRAS, 279, 915

Thé, P. S., de Winter, D., \& Pérez, M. R. 1994, A\&AS, 104, 315

van den Ancker, M. E., Meeus, G., Cami, J., Waters, L. B. F. M., \& Waelkens, C. 2001, A\&A, 369, L17

van den Ancker, M. E., de Winter, D., \& Tjin a Djie, H. R. E. 1998, A\&A, 330, 145

Whittet, D. C. B., Gerakines, P. A., Hough, J. H., \& Shendy, S. S. 2001, ApJ, 547, 872

Whittet, D. C. B., Martin, P. G., Hough, J. H., et al. 1992, ApJ, 386, 562

Yudin, R. V. 2000, A\&AS, 144, 285

Yudin, R. V., \& Evans, A. 1998, A\&AS, 131, 401 